\title{
Kibo: A MIDI Controller with a Tangible User Interface for Music Education
}

\author{
Mattia Davide Amico ${ }^{1}$ and Luca Andrea Ludovico ${ }^{2} \mathbb{1 0}^{\mathrm{a}}$ \\ ${ }^{1}$ Kodaly S.r.l., Via Gian Battista Bazzoni 8, 20123 Milano, Italy \\ ${ }^{2}$ LIM, Laboratorio di Informatica Musicale, Dipartimento di Informatica, Università Degli Studi di Milano, \\ Via Giovanni Celoria 18, 20133 Milano, Italy \\ mdamico@kodaly.app, luca.ludovico@unimi.it
}

Keywords: $\quad$ Music, Education, Tangible User Interfaces, MIDI Controller, Preschool, Primary School, Special Needs.

\begin{abstract}
This paper presents Kibo, a MIDI controller equipped with a simplified tangible user interface. Entirely made of wood, Kibo presents eight geometric extractable solids that can be used to trigger note events and to control musical parameters. In the framework of the cooperation with the Music Informatics Lab of the University of Milan, the distinctive features of the device and their applicability to the field of music education have been investigated. Kibo aims to offer intuitive interaction with music parameters and fosters the acquisition of specific skills in a non-formal learning environment. Benefits are particularly evident for specific target categories of users, including preschool and primary school children and people with both physical and cognitive special needs.
\end{abstract}

\section{INTRODUCTION}

Digital music technology is a broad definition that encompasses digital instruments, computer devices, electronic effects, ad-hoc software, etc., aiming to produce, perform or record music. In this work, we will narrow the field to a specific category of digital equipment, namely musical instruments with a tangible user interface (TUI), employable in a specific domain, namely music education. Some considerations will be easily extensible to other contexts, such as music creativity and computational thinking, where the binomial music-technology can bring a number of benefits. For instance, improving music experience for beginners through simplified interfaces is a way to foster early music education by tearing down the wall of potential frustration and disaffection; and, from the opposite point of view, an educational initiative that uses technology-enhanced tools and gamification processes can draw young users into the sphere of music creativity, also acting as a springboard to formal music education.

The paper is organized as follows: Section 2 will describe the state of the art concerning digital technologies for music education, Section 3 will provide technical details about Kibo, Section 4 will address

\footnotetext{
a (iD https://orcid.org/0000-0002-8251-2231
}

the applicability of Kibo to music learning, in particular for beginners and people with special needs, and Section 5 will draw conclusions.

\section{STATE OF THE ART}

In this section we will review some relevant initiatives based on digital technologies that can help music beginners and people with disabilities in producing music through an embodied approach, with evident benefits (also) for music education.

A research field to mention is the one of technology-enhanced music embodiment. The concept of embodiment implies a corporeal process that enables the link between music as experienced phenomenon and music as physical energy, or the physical environment in general (Leman et al., 2008). Such a concept can be instanced in multiple ways in music education, ranging from immediate music awareness to the acquisition of specific competences. To cite but a few examples, concerning the former aspect, we can mention a social music game where two groups of participants have to collaborate by dancing in a synchronous way (Leman et al., 2009); regarding the latter aspect, it is worth mentioning a computer-based approach to teach tonal harmony to young students who walk within a prepared environment (Avanzini 
et al., 2019b).

This kind of approaches is not merely confined to academic research: there are also commerciallyavailable and successful devices that foster intuitive control of music parameters through the detection of body movements. In this sense, it is worth mentioning Microsoft Kinect (Yoo et al., 2011; Kadakal et al., 2014; Graham-Knight and Tzanetakis, 2015), Nintendo's Wiimote (Kiefer et al., 2008; Reed et al., 2008; Wong et al., 2008), and Leap Motion (Silva et al., 2013; Perdana, 2014; Baratè et al., 2019).

Another approach is based on fiducials. A fiducial marker, or fiducial, is an object placed in the field of view of an image-recognition system which appears in the image produced, employed as a point of reference or a measure. This approach is well exemplified by the reacTable, a digital musical instrument developed by the Music Technology Group at the Universitat Pompeu Fabra in Barcelona, Spain (Jordà, 2010). The reacTable adopts fiducials to generate and control music and sound parameters. This device has a tabletop tangible user interface formed by a round translucent table used as a backlit display. By placing blocks called tangibles on the table, and interfacing with the visual display via the tangibles or fingertips, a virtual modular synthesizer is operated, creating music or sound effects. Other frameworks based on fiducials have been described in literature, such as $\mathrm{Au}-$ dio D-TOUCH (Costanza et al., 2003) and TuneTable (Xambó et al., 2017).

Concerning technology-enhanced TUIs for music, scientific literature describes a number of theoretical approaches, prototypes and available products. A tangible interface - implying something "real", "concrete" - offers a physical way to interact with music and sound parameters. Most traditional musical instruments are played through this kind of interaction, but the advent of digital technologies paved the way to innovative and original approaches. To cite but a few, Paradiso et al. (2001) review TUIs based on magnetic tags, Newton-Dunn et al. (2003) describe a way to controls a dynamic polyrhythmic sequencer using physical artifacts, and Schiettecatte and Vanderdonckt (2008) present a distributed cube interface based on interaction range for sound design. Kibo, the device the paper is focused on, implements a music-oriented tangible interface, so this subject will be further discussed below.

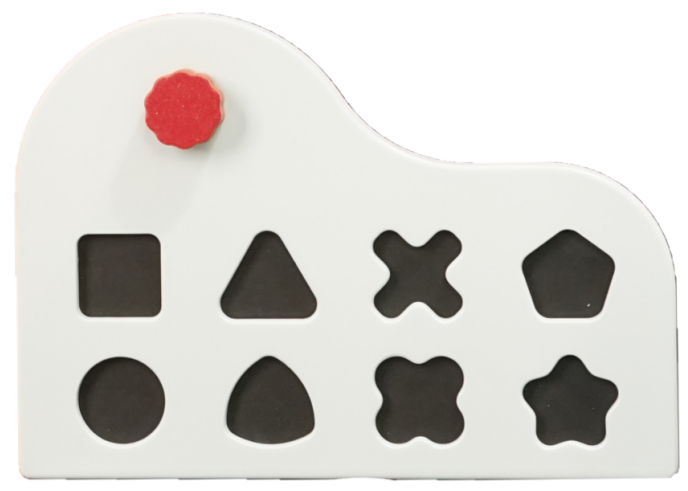

Figure 1: The interface of Kibo.

\section{KEY FEATURES OF THE DEVICE}

Kibo is an interactive instrument produced by Kodaly S.r.l., entirely made of wood, with a simplified tangible interface that embeds a number of controls. The goal of Kibo is to translate musical parameters into geometric shapes through the development of visual, tactile, auditive and mnemonic capacities. From this point of view, the most relevant feature is the presence of eight geometric extractable solids; in addition, the interface includes a multi-function rotating knob (see Figure 1). The device also integrates an accelerometer.

From a technical point of view, Kibo is a MIDI-compatible controller that can communicate with other MIDI devices (e.g., synthesizers and sequencers) thanks to the MIDI-over-Bluetooth protocol (Bartolomeu et al., 2006). MIDI, standing for $\mathrm{Mu}-$ sical Instrument Digital Interface, is a standard and well-documented communication protocol that supports the connection of a wide variety of electronic musical instruments, computers, and related audio devices for playing, editing and recording music.

Kibo triggers MIDI events through its tangibles and knob. Specifically, each tangible object generates Note On and Note Off messages sent over the base channel of the device. These messages are produced both when tangibles are inserted into / extracted from the base, and when they are pressed / released if already in place. Besides, after the activation of a note, each tangible is able to recognize pressure variations, transformed into Polyphonic Aftertouch messages. The rotating knob can detect: a prolonged pressure, used to turn Kibo on/off; a single click, associated with Control Change 119; a double click, associated with Control Change 118; clockwise and counterclockwise rotations, mapped onto step-by-step increasing and decreasing values of Control Change 
117 or sending Program Change messages. ${ }^{1}$ In this way, the knob is a multi-function control that allows to scroll among different choices, set and confirm values.

Kibo can be used also inside a standard music class, namely in conjunction with traditional musical instruments, given the availability of a MIDI synthesizer. This aspect is not particularly limiting, as a PC or even a smartphone can host a dedicated app to parse MIDI messages and produce sound.

However, a characteristic particularly relevant in the field of music education is the possibility to connect up to 7 Kibo devices to the same receiver, thus supporting collaboration within the same musical performance. If a single Kibo in itself would be sufficient to achieve cooperative performances by letting multiple users act on different tangibles (see Figure 2 ), this aspect opens new scenarios, such as creating a Kibo ensemble and playing different parts on up to 7 federated Kibos (see Figure 3). The limitation of maximum 7 independent devices is due to the limited bandwidth supported by MIDI-over-Bluetooth, especially in a context where MIDI choking is a serious risk. $^{2}$

In order to make Kibo a ready-to-use instrument, it can be directly connected to an iPhone, iPad or a Mac thanks to a dedicated app, thus creating a minimal controller-synthesizer MIDI chain. In addition, the app provides a fast way to configure some music parameters (e.g., the current timbre, the musical scale associated to tangibles, etc.) and set the operating mode (see Section 3.2).

\subsection{Tangibles}

The main control over music parameters in Kibo is realized through a set of 8 easily-recognizable tangibles, shown in Figure 4. Each object has a different shape fitting in a single slot. From a physical point of view, tangibles present symmetry properties so that they can be rotated and flipped before being inserted in their slots. They have a magnetic core, consequently they can be also stacked one on top of the other.

The body of Kibo contains a multi-point pressure sensor that allows to detect the insertion and removal of tangibles. The characteristics of the sensor make the instrument both extremely sensitive and very resistant. Concerning the former aspect, it is sufficient

\footnotetext{
${ }^{1}$ Details about the MIDI protocol and a complete reference guide for its messages are available at https://www. midi.org/.

${ }^{2}$ MIDI choking occurs when MIDI data are sent at a rate exceeding what the channel is capable of transmitting.
}

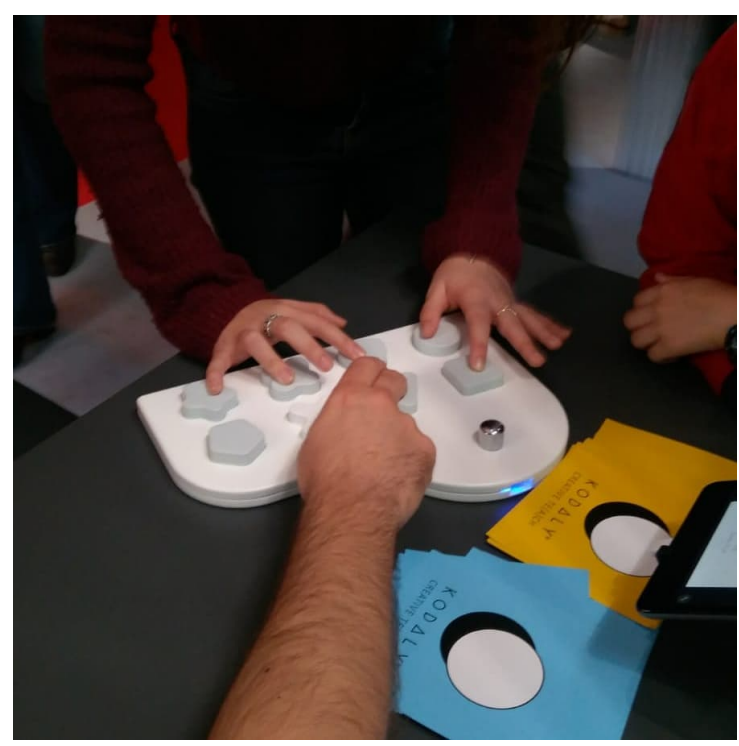

Figure 2: Multiple users playing the same Kibo.

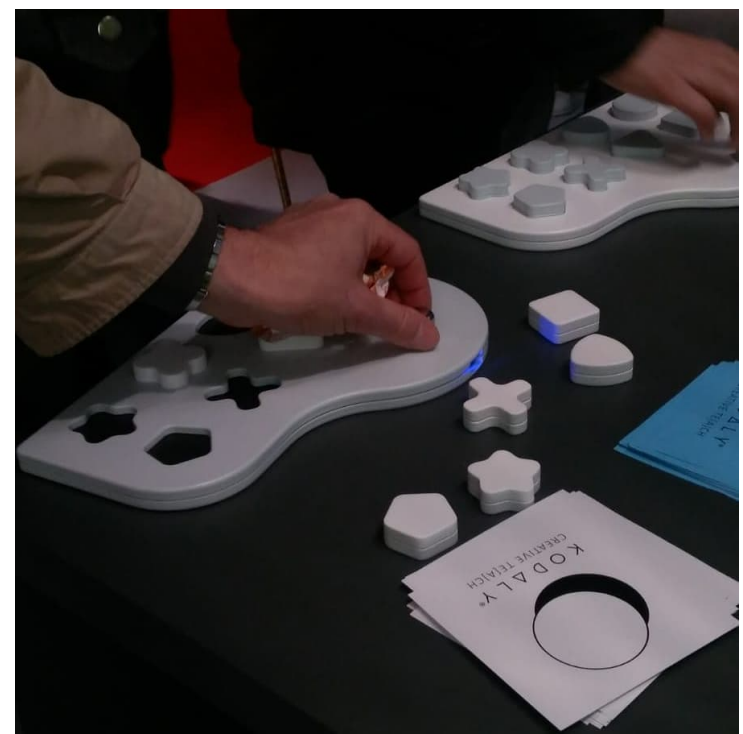

Figure 3: Distributed performance over different Kibos.

to bring a tangible closer to the body to trigger a reaction; similarly, the gentle touch of fingers over an already plugged tangible is recognized as a pressure variation. Concerning robustness, Kibo has been designed to tolerate strong physical stresses, like fists and bumps.

A distinctive feature, supported by the MIDI protocol since its birth but uncommon in MIDI controllers, is the possibility to detect pressure variations over tangibles.

Potentially, different colors can further remark the difference among tangibles and reinforce connections with pitches or musical instruments. It is worth mentioning that color has been often employed in music 
education for beginners (Rogers, 1991; Kajs et al., 1998; Poast, 2000; Oshima et al., 2002; Kuo and Chuang, 2013). In some cases, color information is so useful that even traditional instruments embed it to help musicians in identifying pitches. A well-known example is provided by the harp, whose strings are color-coded for quick reference: all $\mathrm{C}$ strings are red and $\mathrm{F}$ strings are black or dark blue.

\subsection{Operating Modes}

As mentioned before, Kibo is basically a MIDIcompatible controller. Consequently, its operating modes can be extended through ad-hoc applications that associate MIDI messages launched by the device to music and sound production.

In origin, tangibles have been conceived as event triggers, and the knob as a scrolling selector. Such a vision has inspired three operating modes, so far:

1. Advanced Keyboard. - In this scenario, apparently the most obvious one, tangibles are mapped onto pitches. Associations between shapes and notes can be customized. In this way, the device is not limited to the $\mathrm{C}$-major scale but it support key changes, other scale models, unusual note positions, etc. Through software processing of MIDI messages, a single pressed key could also produce custom chords or arpeggios. The metaphor of a keyboard controller is further extended by the availability of the aftertouch effect, namely the possibility to change the pressure on the key after the note attack;

2. Percussive Instrument. - As a variation of the previous scenario, tangibles are mapped onto single percussive instruments. The pressure sensor, resistant but also extremely sensible, allows effects ranging from hard mallet beats to delicate brush rubbing. From an educational point of view, such an operating mode limits the number of music parameters to be managed (e.g., there is no note release and no melodic contour), thus making the performance more intuitive for beginners;
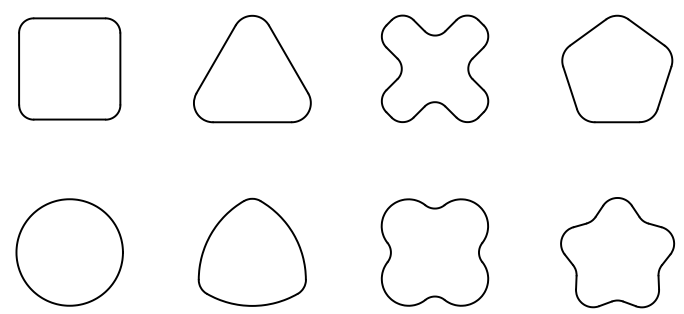

Figure 4: The shapes of Kibo tangibles.
3. DJ Console. - The third scenario consists in using Kibo as a controller for already available music loops. In this case, tangibles are associated with independent but synchronized tracks within a multi-track environment. When tangibles are inserted, the corresponding tracks are activated; when they are removed, tracks are muted (but still running, so as to preserve global synchronization). This is a completely different form of interaction with music content, particularly suitable to engage users who are not able or do not wish to create their own music.

Thanks to the adoption of MIDI, possibilities are endless. Other operating modes could be easily implemented via software, by reading MIDI messages and giving them other meanings, even extra-musical ones. Examples may include the use of tangibles to select and rearrange the sections of a score, to quickly provide answers in a musical quiz, or to animate an object or a character in a music-oriented video game.

\section{APPLICATIONS FOR MUSIC EDUCATION}

Kibo is a hardware device which has potential applications in multiple fields, from pure entertainment to music creativity. In this context, we are particularly interested in scenarios dealing with music formal and non-formal education, a category that ranges from introductory courses for preschool children to the inclusion of people with disabilities in music performances.

\subsection{Music Education in Preschool and Primary School}

A first scenario where Kibo can present advantages with respect to traditional approaches and methodologies is early music education.

Kibo allows to investigate basic music parameters, one by one or combining them in multiple ways. For example, the keyboard mode allows to explore melodic (pitch, intensity, scale models, etc.) and timbral aspects; the percussive operating mode encourages rhythm awareness (tempo, note lengths, accents, etc.); the simultaneous use of multiple tangibles allows to explore harmonic aspects as well. Thanks to suitable settings or ad-hoc software applications, user experience can be customized in order to respond to specific educational needs. For instance, several methods aiming to develop musical skills and teach 
theoretical concepts to very young children emphasize the adoption of the pentatonic scale, which plays a significant role, e.g., in Orff, Kodály, and Waldorf methodologies at an early stage (Landis and Carder, 1972). In this context, Kibo tangibles can be mapped onto the pitches of the pentatonic scale, moving towards major/minor scale models at a more advanced stage.

Another application, linked to the use of clearly recognizable shapes to identify pitches, is the introduction of an alternative music notation for preschool or primary school beginners. Scientific literature describes many initiatives, including binary systems (Bukspan, 1979), LEGO building blocks (Baratè et al., 2017), 3D printed models (Avanzini et al., 2019a), and a number of graphical notations invented by children themselves (Upitis, 1990). Kibo's shapes, potentially reinforced by color associations, let the facilitator describe music tunes through drawings, which is particularly useful for children unable to read or affected by cognitive impairments.

Finally, Kibo can be applied to the development of computational thinking, thanks to the use of patterns whose musical meaning is made explicit. By associating pitches, or note sequences, or chords, or instrumental patches to specific tangible layouts, a relationship among shape, position and musical meaning can be established. Such a structure, after a suitable explanation by the facilitator, can be practiced alone (individual exploration, self-regulation, etc.) or in group (cooperatively, in a peer-to-peer review context, etc.). Moreover, associations can be modified, in order to foster reasoning and abstraction skills.

For the sake of clarity, let us present a simple use case. With Kibo working in the keyboard operating mode and configured to follow C-major grades, the sequence of tangibles can be altered with respect to the ascending diatonic scale $(\mathrm{C}, \mathrm{D}, \mathrm{E}, \mathrm{F}, \mathrm{G}, \mathrm{A}, \mathrm{B}$, C), so as to associate the same (transposed) interval - e.g., a third or a fifth - to vertically paired tangibles. In this way, the user learns to associate an interval model to a physical layout, and simultaneously develops harmonic awareness. After a number of training sessions, the associated interval can change: on one side, the palette of known intervals is extended; on the other, the action "press two vertically-aligned tangibles" is no more linked to a predefined interval, but means "repeat the current interval model on another scale grade", or, in other terms, "transpose the current interval".

\subsection{Music Education for People with Special Needs}

In literature, the term enabling technology identifies a technology that alleviates the impact of disease or disability. Four categories of enabling technology were pointed out by Hansson (2007):

- Therapeutic - Restore the original biological function that has been lost or prevent further losses;

- Compensatory - Fully or partially replace a lost biological function by a new function of a general nature;

- Assistive - Allow users to perform a task or an activity despite an uncompensated disability or lack of function;

- Universal - Intended for general use.

When focusing on education, Kibo can be considered an assistive technology from multiple points of view. It becomes therapeutic when used in a rehabilitation context, for both cognitive and physical impairments. In this case, music expression can be seen as a way to push the limits, e.g. as an engaging goal to achieve through embodiment. The attribution of a physical identity to music parameters eases the use of the instrument for visually impaired people as well as people with dysfunctions like autism. The educational aspect may concern both the acquisition of music awareness and the improvement of extra-musical abilities, such as motor or cognitive ones.

Kibo can also be a compensatory enabling technology, since its fine sensing capabilities let it detect even small movements. For instance, it is possible for a quadriplegic user to hit drums by slight pressure variations on tangibles. Similarly, the unique shape of each tangible make it simple for a blind or visually impaired (BVI) user to trigger music events, whereas many traditional instruments are not accessible without ad-hoc additions. In music education the availability of a simplified, but expressive music interface is a key aspect to engage this category of users.

Finally, Kibo is also an assistive enabling technology, since it fosters creativity and encourages participation in musical performances also in case of uncompensated disabilities and disorders. For example, the "DJ console" operating mode is a way to produce potentially complex musical structures and interactions even in case of serious physical or cognitive impairments. 


\subsection{Advanced Music Education}

Among potential applications in the educational field, it is worth mentioning the use of Kibo as a simple but powerful device to study and put into practice a number of concepts typical of sound and music computing.

Since Kibo is a MIDI controller, a first case study is learning the theoretical principles and practical rudiments of the MIDI protocol, with the possibility to deepen non-trivial aspects such as distributed musical performances, articulated MIDI chains, MIDI choking in presence of fine-granularity continuous controllers, etc.

Moreover, even if Kibo is basically a controller, sound production is demanded to a synthesizer, which in turn can be controlled via Kibo. Exploring different MIDI programs, i.e. instrumental patches and sound banks, can stimulate the study of sound-synthesis models, such as sound sampling and table lookup methods. Other advanced subjects can be explored, such as the SoundFont technology that uses samplebased synthesis to play MIDI events.

Finally, the features of Kibo can arise exquisitely technical discussions, concerning issues such as the resolution of MIDI choking and MIDI-over-Bluetooth bandwidth limitations, or the characteristics of a pressure sensor capable of detecting fine variations and withstanding strong stresses as well.

Needless to say, this kind of approach is suitable mainly for students with a musical and technological background.

From a pedagogical point of view, it is possible to frame Kibo-based activities in the context of the Think-Make-Improve model (Martinez and Stager, 2013), by recording students' performances, e.g. through a MIDI sequencer connected to Kibo, and inviting them to self-reflect about their actions.

\section{CONCLUSIONS}

Kibo is a MIDI controller presenting a simplified tangible interface that conjugates research in the artistic, scientific and social field in order to foster music expression and creativity. When properly integrated within a pedagogical method, Kibo can promote music education through technology so as to facilitate cooperation, social inclusion and cultural exchange.

Concerning future work, Kibo requires a thorough experimentation in specific educational contexts. From this point of view, we are planning adhoc educational labs in cooperation with Fondazione Clerici Milano, addressing specific user categories, specifically BVI people and learners with cognitive special needs.

\section{REFERENCES}

Avanzini, F., Baratè, A., and Ludovico, L. A. (2019a) $3 \mathrm{~d}$ printing in preschool music education: Opportunities and challenges. Qwerty - Open and Interdisciplinary Journal of Technology, Culture and Education, 14(1):71-92.

Avanzini, F., Baratè, A., Ludovico, L. A., and Mandanici, M. (2019b). A computer-based approach to teach tonal harmony to young students. In Lane, H., Uhomoibhi, J., and Zvacek, S., editors, Proceedings of the 11th International Conference on Computer Supported Education (CSEDU 2019), volume 1, pages 271-279. SCITEPRESS - Science and Technology Publications, Lda.

Baratè, A., Ludovico, L. A., and Malchiodi, D. (2017). Fostering computational thinking in primary school through a LEGO®-based music notation. Procedia Computer Science. Knowledge-Based and Intelligent Information and Engineering Systems: Proceedings of the 21st International Conference, KES 2017, 6-8 September 2017, Marseille, France, 112:1334-1344.

Baratè, A., Ludovico, L. A., and Oriolo, E. (2019). Investigating embodied music expression through the Leap Motion: Experimentations in educational and clinical contexts. In McLaren, B. M., Reilly, R., Uhomoibhi, J., and Zvacek, S., editors, Computer Supported Education - 10th International Conference, CSEDU 2018 Funchal, Madeira, Portugal, March 15-17, 2018, Revised Selected Papers, volume 1022 of Communications in Computer and Information Science, pages 532-548. Springer International Publishing.

Bartolomeu, P., Fonseca, J., Rodrigues, P., and Girao, L. (2006). Evaluating the timeliness of Bluetooth ACL connections for the wireless transmission of MIDI. In 2006 IEEE Conference on Emerging Technologies and Factory Automation, pages 46-53.

Bukspan, Y. (1979). Introduction of musical literacy to children by means of a binary system of music notation: An experimental study. Bulletin of the Council for Research in Music Education, pages 13-17.

Costanza, E., Shelley, S. B., and Robinson, J. (2003). Introducing audio D-TOUCH: A tangible user interface for music composition and performance. In Proc. of the 6th Int. Conference on Digital Audio Effects (DAFX03), London, UK, September 8-11, 2003.

Graham-Knight, K. and Tzanetakis, G. (2015). Adaptive music technology using the Kinect. In Proceedings of the 8th ACM International Conference on PErvasive Technologies Related to Assistive Environments, pages $1-4$.

Hansson, S. O. (2007). The ethics of enabling technology. Cambridge Quarterly of Healthcare Ethics, 16(3):257-267.

Jordà, S. (2010). The reactable: tangible and tabletop music performance. In CHI'10 Extended Abstracts on 
Human Factors in Computing Systems, pages 29892994.

Kadakal, Y., Kivrak, H., and Kose, H. (2014). Kinect based interactive music application for disabled children. In 2014 22nd Signal Processing and Coтmunications Applications Conference (SIU), pages 453456. IEEE.

Kajs, L. T., Alaniz, R., Willman, E., and Sifuentes, E. (1998). Color-coding keyboard functions to develop kindergartners' computer literacy. Journal of Computing in Childhood Education.

Kiefer, C., Collins, N., and Fitzpatrick, G. (2008). Evaluating the wiimote as a musical controller. In Proceedings of the 2008 International Computer Music Conference, ICMC 2008, Belfast, Ireland, August 24-29, 2008.

Kuo, Y.-T. and Chuang, M.-C. (2013). A proposal of a color music notation system on a single melody for music beginners. International Journal of Music Education, 31(4):394-412.

Landis, B. and Carder, P. (1972). The eclectic curriculum in American music education: Contributions of Dalcroze, Kodaly, and Orff.

Leman, M., Demey, M., Lesaffre, M., van Noorden, L., and Moelants, D. (2009). Concepts, technology, and assessment of the social music game "sync-in-team'. In 2009 International Conference on Computational Science and Engineering, volume 4, pages 837-842.

Leman, M. et al. (2008). Embodied music cognition and mediation technology. MIT press.

Martinez, S. L. and Stager, G. (2013). Invent to learn. Making, Tinkering, and Engineering in the Classroom. Torrance, Canada: Construting Modern Knowledge.

Newton-Dunn, H., Nakano, H., and Gibson, J. (2003). Block jam: a tangible interface for interactive music. Journal of New Music Research, 32(4):383-393.

Oshima, C., Miyagawa, Y., and Nishimoto, K. (2002). Coloring-in piano: A piano that allows a performer to concentrate on musical expression. In Proceedings of the 7th International Conference on Music Perception and Cognition, Sydney 2002, pages 707-710.

Paradiso, J. A., Hsiao, K.-y., and Benbasat, A. (2001). Tangible music interfaces using passive magnetic tags. In Proceedings of the 2001 conference on New interfaces for musical expression, pages 1-4.

Perdana, I. (2014). Teaching elementary school students new method of music performance with Leap Motion. In 2014 International Conference on Virtual Systems \& Multimedia (VSMM), pages 273-277. IEEE.

Poast, M. (2000). Color music: Visual color notation for musical expression. Leonardo, 33(3):215-221.

Reed, A., Dons, K., Thompson, M. R., and Tervo, T. (2008). Wiimote music-towards an intuitive gestural interface grounded in a theory of learning schemas. In 3rd International Haptic and Auditory Interaction Design Workshop, pages 16-17.

Rogers, G. L. (1991). Effect of color-coded notation on music achievement of elementary instrumental students. Journal of Research in Music Education, 39(1):64-73.

Schiettecatte, B. and Vanderdonckt, J. (2008). Audiocubes: a distributed cube tangible interface based on interac- tion range for sound design. In Proceedings of the 2nd international conference on Tangible and embedded interaction, pages 3-10.

Silva, E. S., de Abreu, J. A. O., de Almeida, J. H. P., Teichrieb, V., and Ramalho, G. L. (2013). A preliminary evaluation of the Leap Motion sensor as controller of new digital musical instruments. Recife, Brasil, pages 59-70.

Upitis, R. (1990). Children's invented notations of familiar and unfamiliar melodies. Psychomusicology: A Journal of Research in Music Cognition, 9(1):89.

Wong, E. L., Yuen, W. Y., and Choy, C. S. (2008). Designing Wii controller: a powerful musical instrument in an interactive music performance system. In Proceedings of the 6th International Conference on Advances in Mobile Computing and Multimedia, pages 82-87.

Xambó, A., Drozda, B., Weisling, A., Magerko, B., Huet, M., Gasque, T., and Freeman, J. (2017). Experience and ownership with a tangible computational music installation for informal learning. In Proceedings of the Eleventh International Conference on Tangible, Embedded, and Embodied Interaction, pages 351360.

Yoo, M.-J., Beak, J.-W., and Lee, I.-K. (2011). Creating musical expression using Kinect. In Proceedings of the International Conference on New Interfaces for Musical Expression, 30 May - 1 June 2011, Oslo, Norway, pages 324-325. 